Brit. Heart F., 1966, 28, 388.

\title{
Localization of Atheroma: A Theory Based on Boundary Layer Separation
}

\author{
J. A. FOX AND A. E. HUGH* \\ From the Department of Civil Engineering, University of Leeds, and The North Staffordshire Royal Infirmary, \\ Stoke-on-Trent
}

Current reports of research into the problem of atheroma deal largely with abnormalities and peculiarities of lipid metabolism. As atheromatous plaques are rich in lipids, and can be produced in most experimental animals by a high cholesterol diet, it is reasonable to suppose that atheroma and lipid metabolism are related. There will, however, be some disagreement with Hess (1964) who stated that "atherosclerosis is now rather generally accepted to be a disorder of lipid metabolism", because there are several features of the disease that are not explained in terms of lipid disturbance.

The main alternative theory is that atheroma is the result of intravascular clotting or thrombosis. This was originally expounded by Rokitansky in a paper in 1844, and it has been supported by several authors since, in particular by Duguid (1955) and Osborn (1963) in this country. The two theories mentioned have appeared to compete with one another for acceptance, and it has not hitherto been possible to suggest a way in which they might be related.

The development of atheroma is undoubtedly related to hypertension as well as to lipid disturbance (Corcoran et al., 1956; Schettler, 1961). Two explanations of this relationship have been offered: Masson et al. (1958) believed that hypertension produced widespread vascular damage which facilitated lipid deposition, while Page (1954) suggested that hypertension would lead to an increased transarterial filtration of some of the contents of the circulating fluid, either plasma lipids (Page) or protein (Duncan, Cornfield, and Buck, 1962), which would initiate the local formation of atheroma.

Any explanation of atheroma must account for

Received June 23, 1965.

*Formerly at the Department of Radiodiagnosis, University of Leeds. the focal nature of the lesions and their predilection for certain areas of the arterial system, notably curved segments and the mouths of branch vessels. Though local mechanical strain was for long believed to account for this, the alternative view, that flow disturbances would affect these areas, has received more recent support (Texon, 1960; Mitchell and Schwartz, 1965). Whereas Texon believed that the intima might become "sucked off" the media due to the generation of low pressures within the lumen by local velocity alterations, the latter support the concept that atheroma is due to thrombosis, precipitated by local turbulence, which would damage either the wall itself or the constituents of the blood.

The use of the term "turbulence" implies that extra motion is being generated; it is conventional to believe, however, that thrombosis or clotting is facilitated by stasis rather than by excessive movement, and it is doubted whether turbulence itself is the cause of intra-arterial thrombus formation.

It has not been widely believed that zones of stasis might occur in the arterial system. In a fluid system, however, it is possible for well-defined static zones to form despite quite high velocities near-by, due to a process known as "boundary layer separation". There is a striking similarity between the regions where this occurs and the sites of predilection of atheroma. We believe that static zones occur in the arterial system, which might allow the interaction of platelets and fibrin to form a mesh in which lipid particles become trapped, and that this becomes organized to form a plaque of atheroma as envisaged by Duguid.

The process of boundary layer separation and the factors that influence it are described, and it will be suggested how a number of the factors that are known to be related to atherogenesis might affect 388 
between the "lipid" and the "thrombotic" theories previously mentioned, and it may well form a basis for a rational approach to the prevention if not the treatment of the disease.

\section{THE BOUNDARY LAYER}

When flow occurs over any surface, the fluid adjacent to it is brought to rest because of the frictional stresses exerted upon the fluid by the surface. Layers further from the surface also have their velocity reduced by these stresses, the reduction being less the further the layer is situated from the boundary. A typical velocity distribution produced in this manner is shown in Fig. 1.

The boundary layer thickness is usually assumed to be equal to that distance from the boundary at which the local velocity ' $u$ ' equals $0.99 U$, where ' $U$ ' is the undisturbed stream velocity.

In a pipe (i.e. a flat boundary rolled into a cylinder), the boundary layers formed at diametrically opposite walls meet at the centre line, and the entire flow is a type of boundary layer flow (except at the entrance of the pipe). In boundary layers, flow may be either laminar or turbulent in nature. If laminar, the velocity distribution is paraboloidal, but if turbulent, the velocity distribution is of an approximately exponential form (more accurately, logarithmic).

Due to these variations in velocity, the fluid near the boundary has low momentum, but the fluid

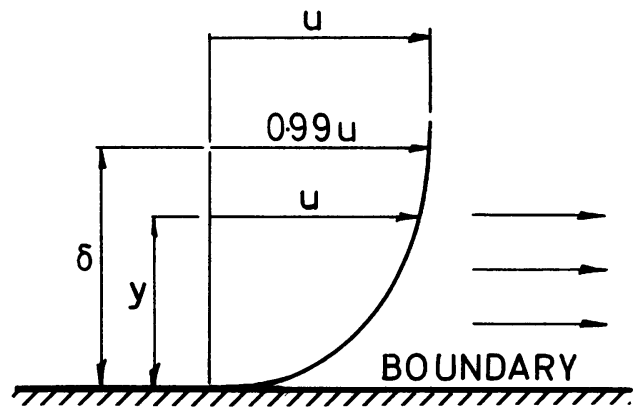

FIG. 1.-Velocity distribution in a boundary layer.

momentum increases rapidly towards the centre of the pipe.

Boundary Layer Separation. Due to its momentum, it is possible for fluid to pass from a region of low pressure into a region of higher pressure (i.e. against a positive pressure gradient). The fluid near a boundary, having low momentum, may rapidly be brought to rest under the action of such a gradient; beyond this stagnation point, its flow will be reversed (Fig. 2).

Because of this reversal near the boundary, a surface forms over which there is no fluid velocity. This is the surface of separation, on one side of which the reversal effects result in the formation of
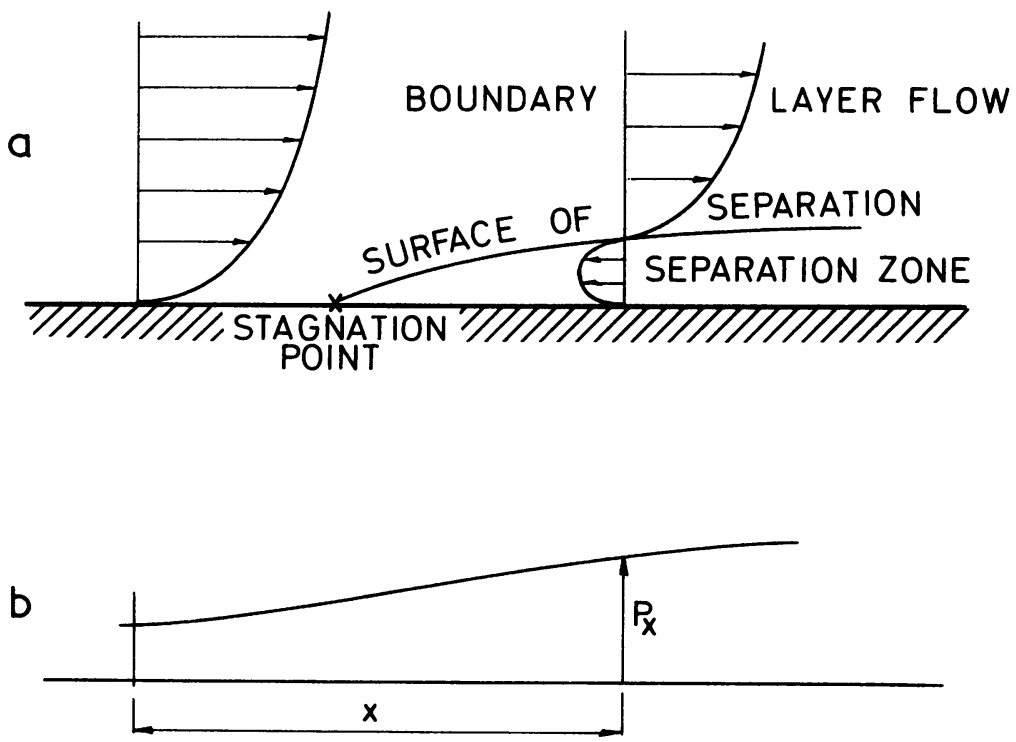

Fig. 2.-(a) Shows the surface of separation and stagnation point of a separated boundary layer and (b) shows the positive pressure gradient that caused the separation. 


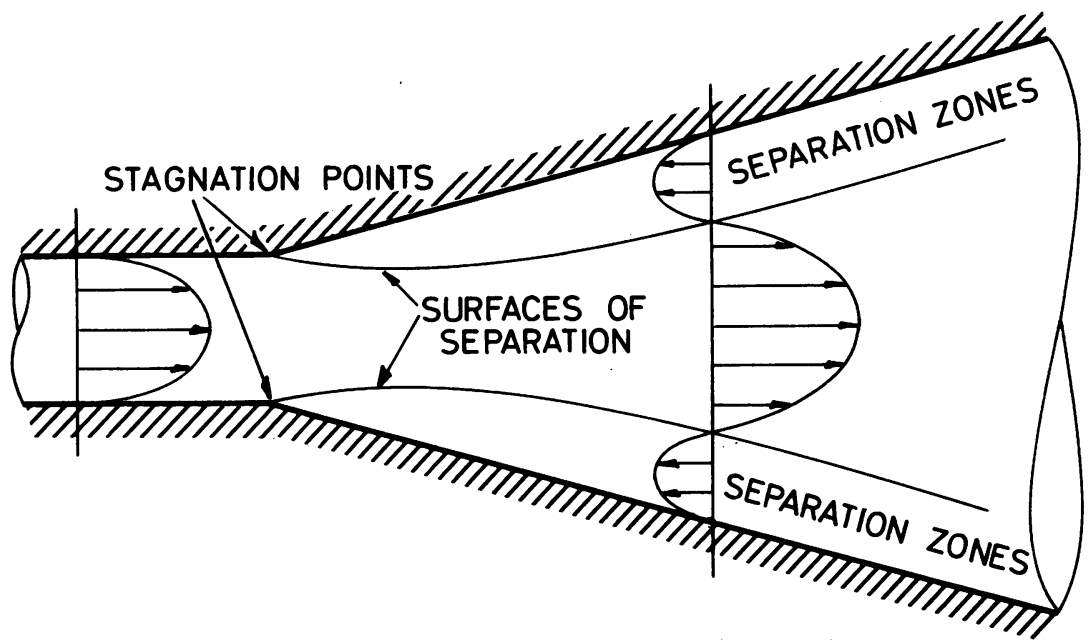

FIG. 3.-Boundary layer separations at divergent duct.

vortices adjacent to the boundary. On the other side, the boundary layer, now separate from the wall, continues its forward motion. Near the wall just beyond the stagnation point the fluid velocities are extremely low, and this allows prolonged contact between the fluid (and its constituents) and the boundary. (This is not a steady process; the surface of separation oscillates due to a type of feedback mechanism.)

Positive pressure gradients can occur (1) in a divergent channel; (2) in a bend; and (3) at a junction.

(1) Divergent Channels. To maintain continuity of flow, average flow velocities must decrease in the

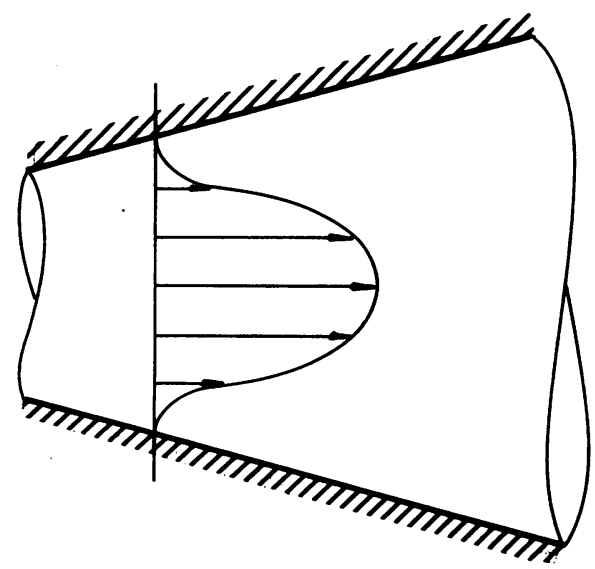

FIG. 4.-Boundary layer just not separating as positive pressure gradient is not large enough. direction of flow (Fig. 3). The energy lost as velocity energy is partly converted into pressure energy (some is lost in turbulent friction). Provided that frictional losses are not excessive, this process can produce a positive pressure gradient, and if this is sufficiently large the boundary layer will separate as illustrated (Fig. 3 ). If the positive pressure gradient is not large enough, the boundary layer will not separate, but the velocity profile will be distorted (Fig. 4).

(2) Curves and Bends (Fig. 5). For flow in a curved path, a force must act in a direction towards the centre of curvature of the flow. Neglecting friction, the pressure in the straight entry section

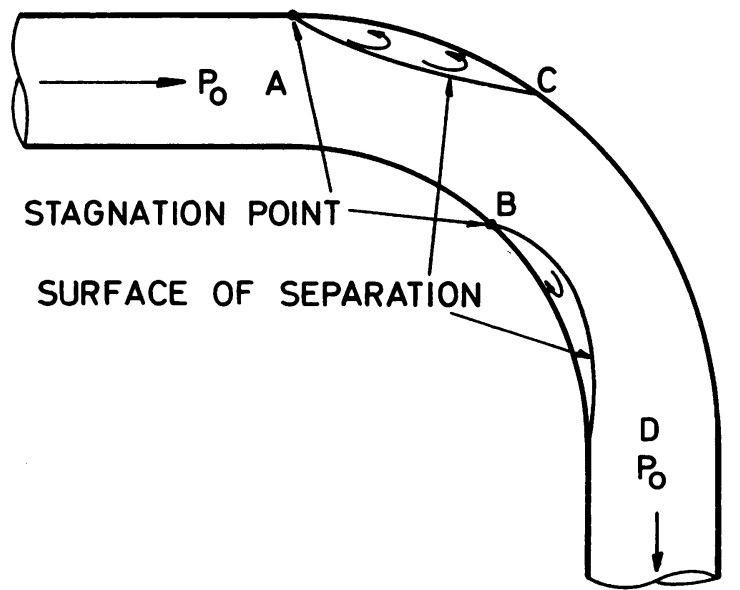

FIG. 5.-Boundary layer separations at bends. 
and in the straight exit section will be the same, i.e. $P_{O}$, and uniform across the vessel cross-section.

At point $B$ the pressure $P_{B}$ will be greater than $P_{O}$, and at $C$ it will be less than $P_{0}$, so that the centrally directed force necessary to cause curvilinear motion can be produced.

The pressure gradients from $A$ to $B$ and $C$ to $D$ must thus be positive, and boundary layer separations will, therefore, occur at these sections as illustrated in Fig. 5.

(3) functions. Referring to Fig. 6, if section 2 is of the same cross-sectional area as section 1, the average velocity at section 2 will be less than at section 1 , because of the outflow through the branch vessel. By a similar argument to that used in considering the divergent vessel problem, the lower velocity at section 2 implies a higher pressure there than at section 1. Slowly-moving fluid near the boundary will tend to have its forward motion reversed if this pressure rise is sufficiently great. The boundary layer on the branch side of the main vessel will be carried off through the branch, but on the opposite wall the boundary layer will be fully exposed to the reversing effect of the pressure rise, which may result in its separation from the wall (see separation A in Fig. 6).

At the mouth of the branch vessel, the fluid has to travel in a curved path; the pressure at the upstream edge of the mouth falls to provide the necessary centrally-directed force required to maintain this curved path. Further along the branch, where the flow is not curvilinear, there will be no transverse pressure gradient. This reversion to a uniform pressure distribution creates a positive-pressure gradient along the upstream wall of the branch, and this is a potent cause of boundary layer separation in this region.

It is thus seen that where a branch vessel is given off, there are two possible sites for boundary layer separation, i.e. in the main vessel opposite and slightly downstream of the axis of the branch, and at the proximal portion of the branch itself. The forces that cause separation are much stronger in the latter.

The above principles apply not only to the case of the right-angled branch but also to junctions of different geometry, including the case of a major vessel dividing into two branches of equal size, provided that the total area of flow increases at such junctions.

Motion within Separation Zone; Effect of Reynolds Number. As boundary-layer separation is a frictional phenomenon, it must be controlled by the
Reynolds number of the flow. If the frictional stresses in the fluid are great in relation to its momentum, then the reverse velocities will be low, i.e. vortex formation will be weak, and the fluid in the separation zone will tend to stagnate. Conversely, if momentum effects dominate the motion, the separated zone will be in a strongly turbulent state.

This ratio of momentum effects to frictional effects is in fact Reynolds number,

$$
R e=\frac{\mathrm{vd}}{\nu} \quad \begin{aligned}
& \mathrm{v}=\text { velocity } \\
& \mathrm{d}=\text { diam. vessel. } \\
& \nu=\text { coeff. kinematic viscosity. }
\end{aligned}
$$

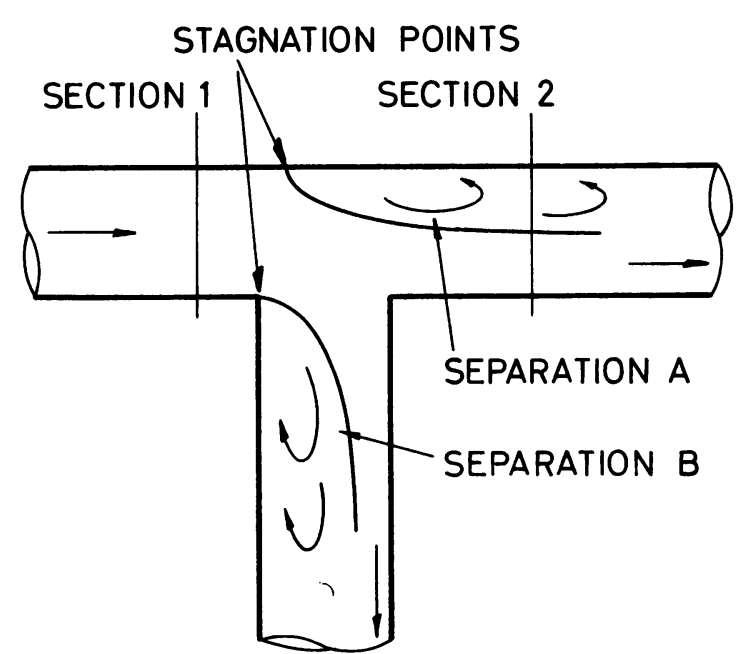

FIG. 6.-Boundary layer separations at junctions.

Flows with low Reynolds numbers have very stagnant separation zones, and flows with high Reynolds numbers have very turbulent separation zones. The factors that cause low Reynolds numbers are low velocity, small diameter, and high viscosity (see expression).

\section{Experimental Demonstration of Phenomena}

It was decided to demonstrate the phenomena described above by using free surface flows in which separations can be demonstrated with greater ease than in bounded flows.

An Ahlborn tank was used in these experiments. This consists of a glass sheet over which sheet flows of water can be produced of any depth and velocity required. Various channel configurations were produced by using strips of aluminium sheet bent to suitable shapes (see Fig. 7-11). 

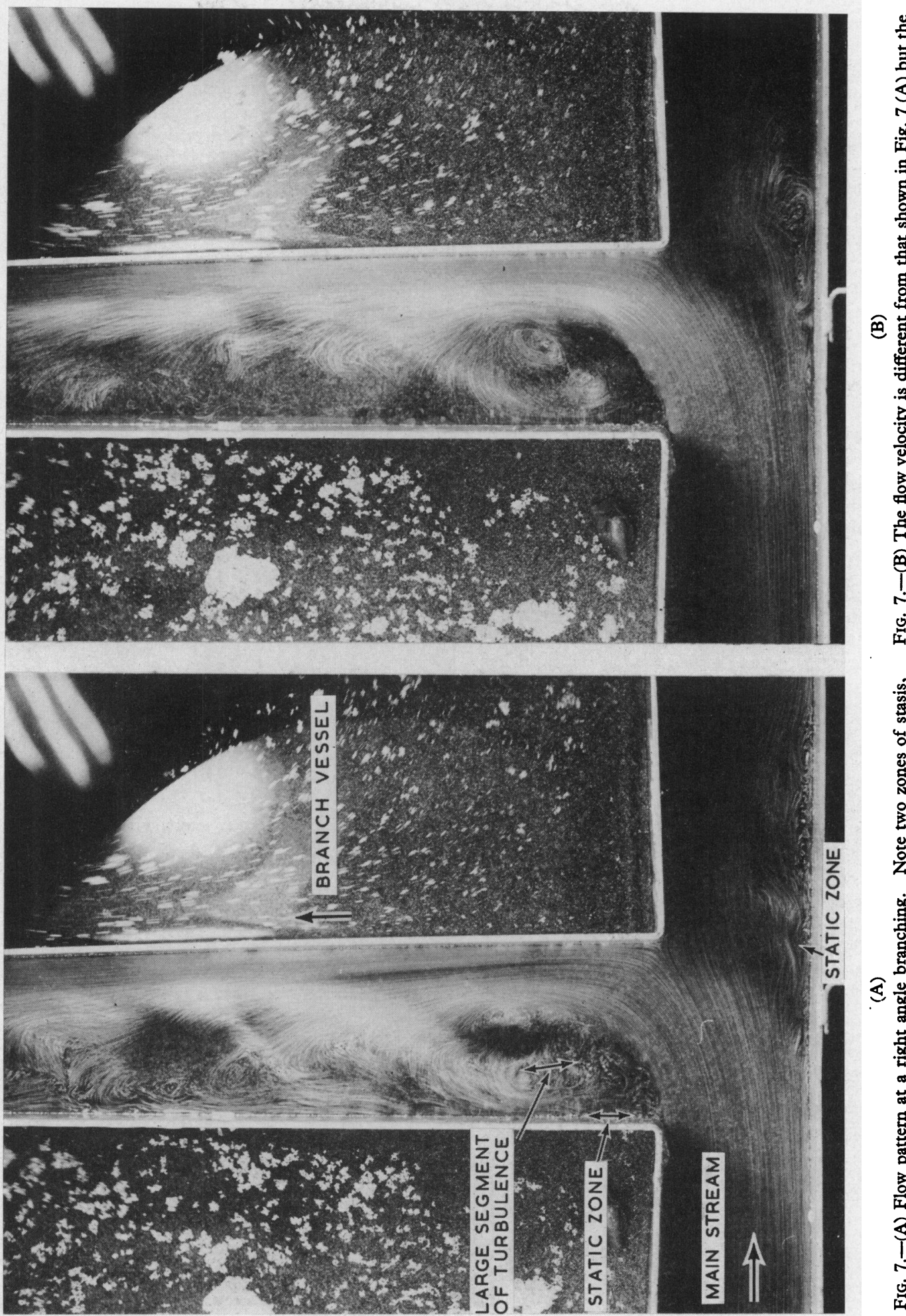

量 

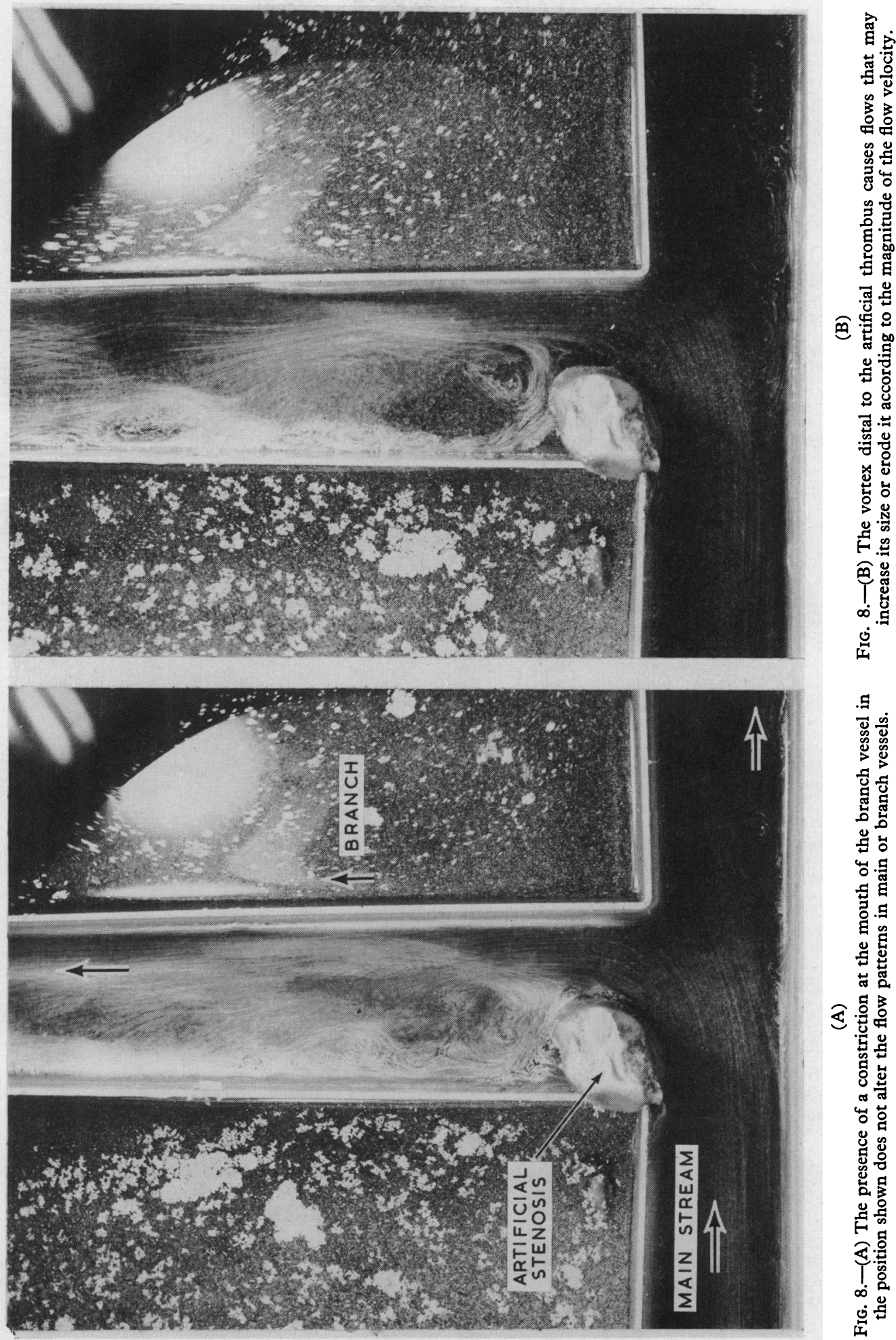

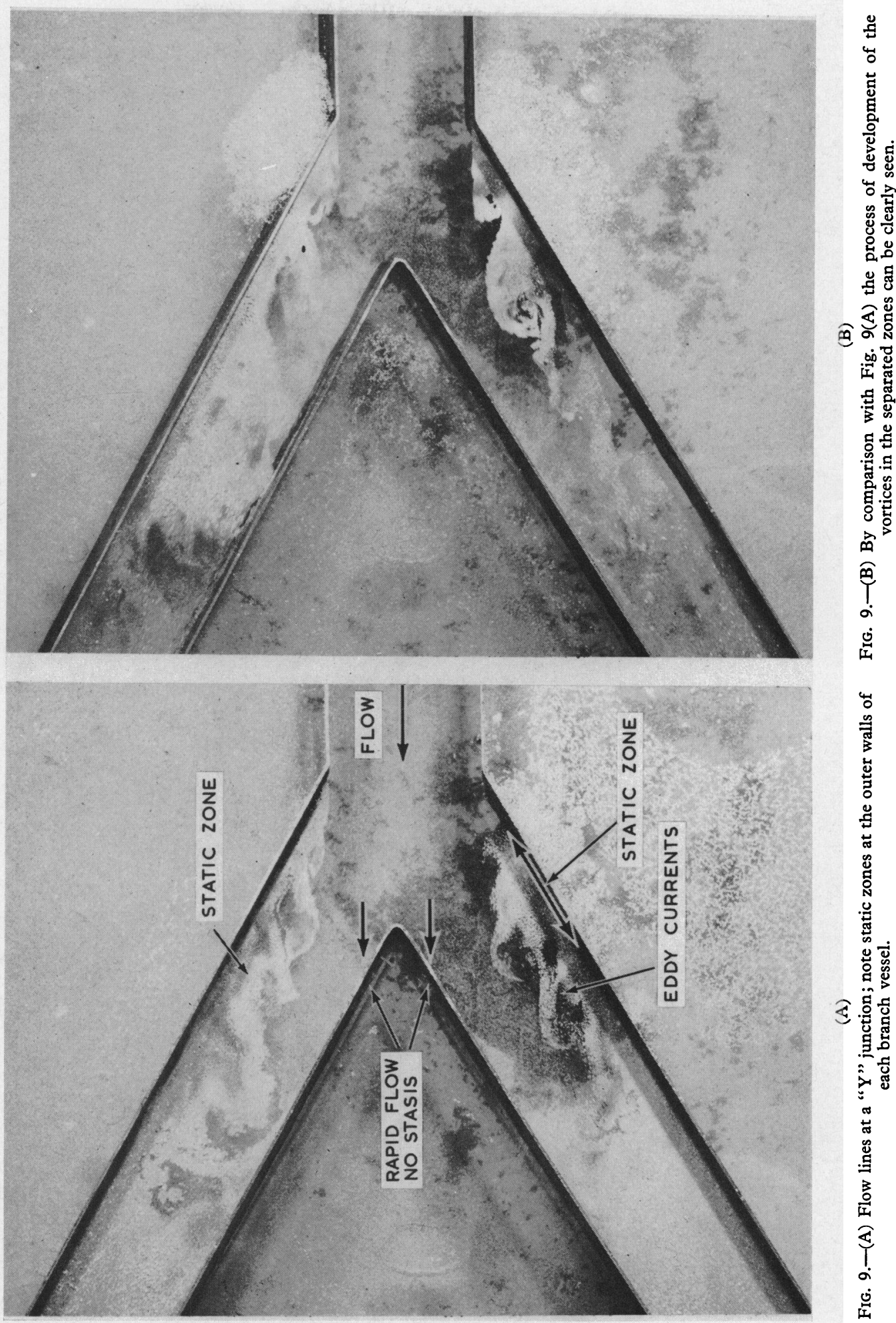

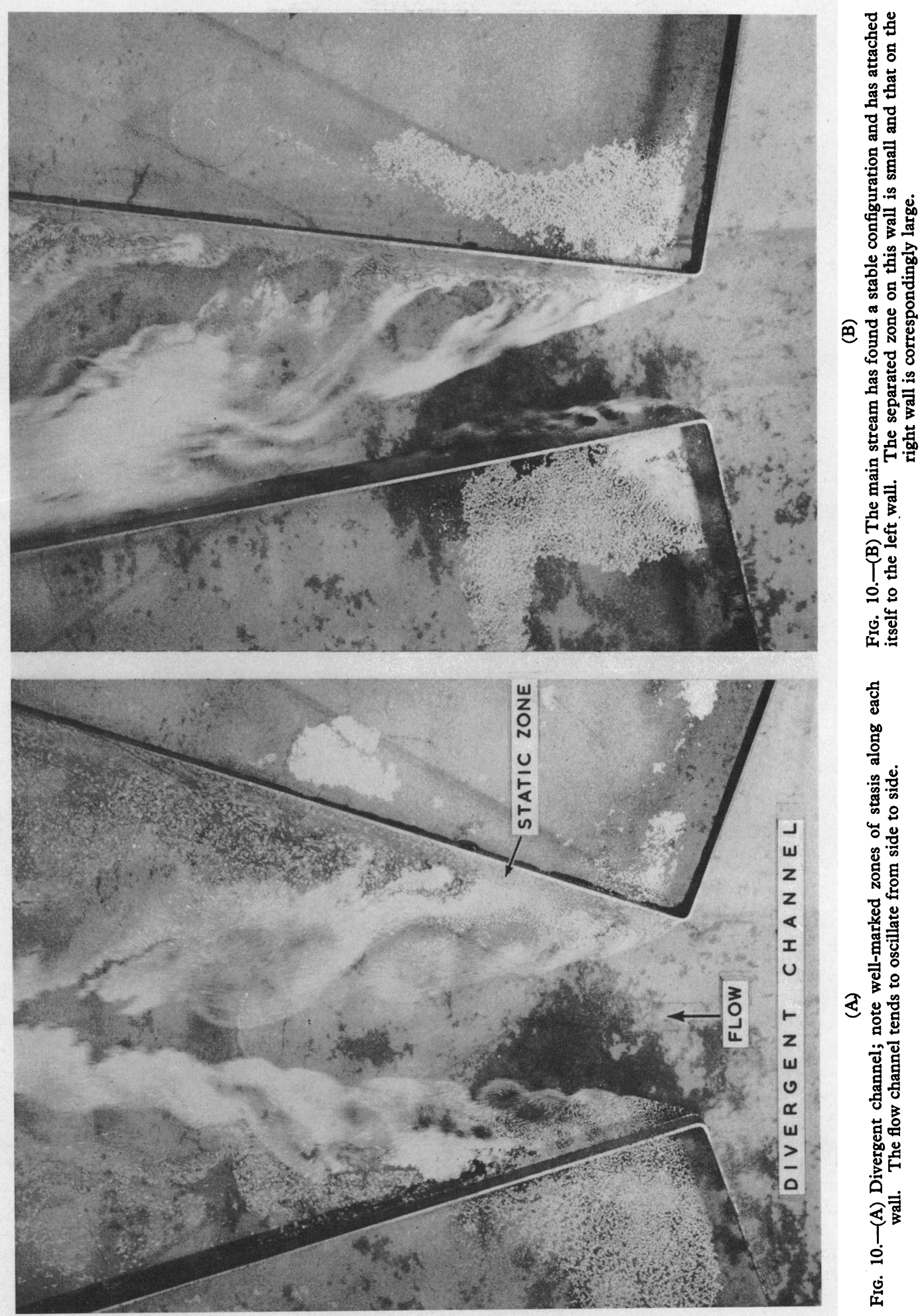

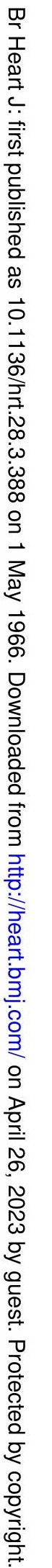




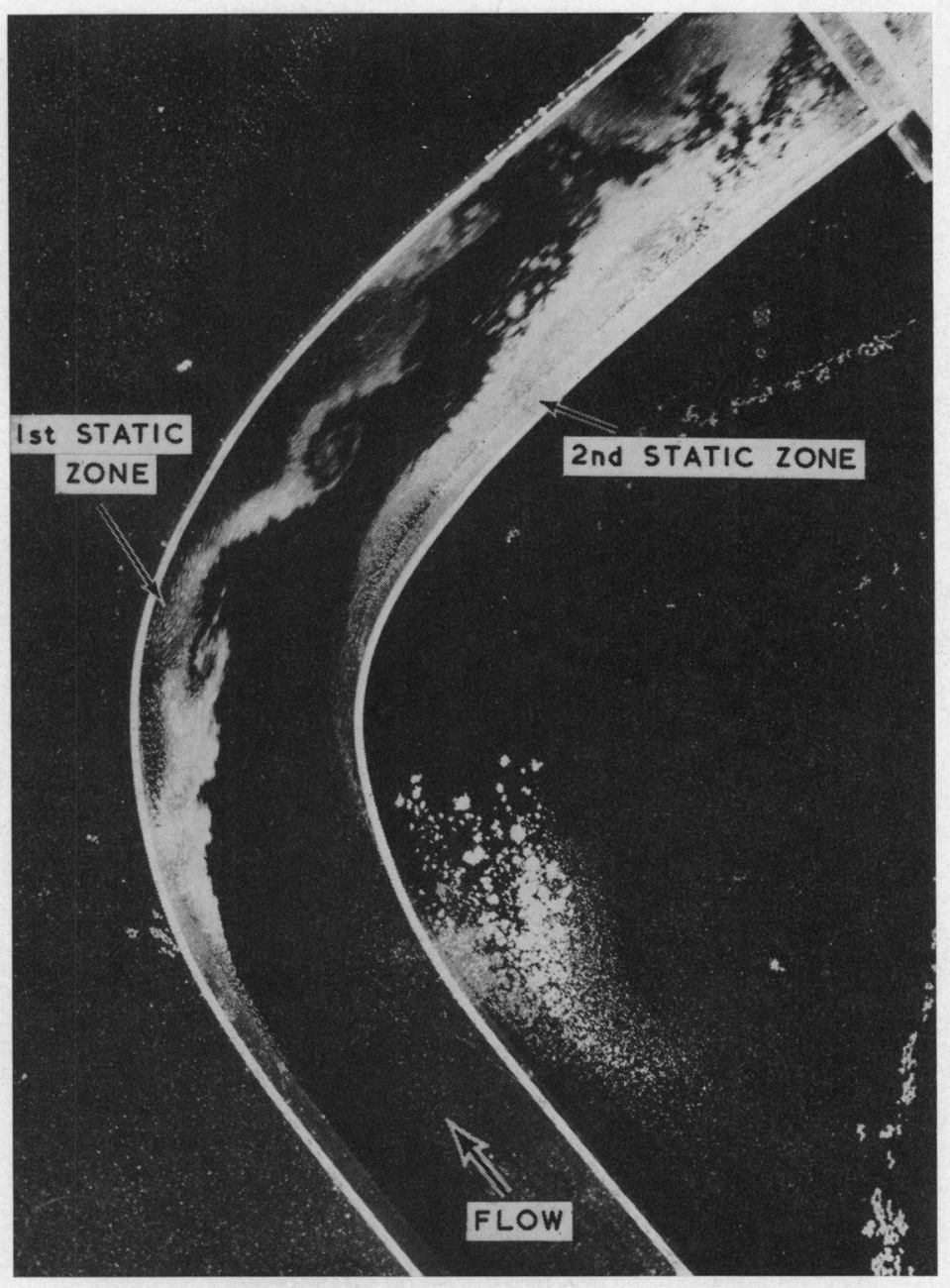

FIG. 11.-At a curved segment, static zones form on opposite walls as shown.

Fine aluminium powder scattered over the water surface was used to reveal the flow patterns, and to facilitate the photography of these patterns.

A wide range of flow velocities was investigated and the theoretical points made in this paper were verified and demonstrated (Fig. 7-11).

It may be felt that an investigation of the phenomena using open channel flows instead of bounded flows renders the experimental work invalid when applied to bounded flows. As surface waves were avoided this possible criticism would not be applicable. The photographs give a picture representing a longitudinal section through the bounded flow problem, i.e. they give a twodimensional section through a three-dimensional flow. 
separate reasons. Either abnormal constituents 'appear in the circulating fluid, such as excessively adhesive platelets which permit formation of a nidus on the vessel wall, which encourages a fibrin meshwork to develop even in the presence of normal amounts of fluid stasis; or, excessive intravascular stasis, due to more marked boundary separation, allows even normal blood constituents to adhere to the vessel wall. In either case the precise nature of the deposit would be determined both by the local shear rate, and by the composition of the circulating fluid. It would be expected that a considerable amount of cholesterol would become enmeshed in the fibrin network if the circulating blood were cholesterol rich, but in neither of the circumstances postulated would an excess of cholesterol be necessary to initiate the process of deposition.

Although well-defined static zones would be likely at arterial mouths at all times, it is thought that in the healthy state the zone would be small and that it would oscillate, both as a natural consequence of the instability of the separation process, and because of physiological fluctuations in flow rates from time to time. These would counteract any tendency to deposition of normal blood contents. Reversal of flow, due to pulsatility, causes boundary layers to form on opposite walls of branch mouths in turn; this might result in the formation of two areas of deposition, or alternatively, could have a cleansing effect on the major site of stasis, so reducing the tendency to deposition and the chance of organization.

From the factors known to control the process of boundary layer separation, it can be predicted that conditions that would give rise to a larger more stably-located area of stasis would be locally reduced velocity and increased blood viscosity. Each of these might become of pathological significance.

In the absence of abnormally-adhesive circulating particles it is possible that variations in viscosity might be the key to atheromatous development.

There are considerable practical difficulties in the accurate measurement of blood viscosity, which prevent this being done as a routine. Several viscometers are available (Harkness, 1963) but no in vivo technique has been devised. Dintenfass has described (1964b) a technique for the fairly rapid estimation of viscosity of blood removed from the circulation, which enables the estimation to be carried out without the need for anticoagulants, but this has yet to be widely adopted. It is found that the thixotropic nature of blood necessitates the employment of a constant rate of shear before comparisons can be made; even this does not provide a standard for comparison with intravascular conditions where shear rates alter constantly.
The evidence available suggests that wide variations in blood viscosity are possible, in various pathological states, such as congestive failure and coronary disease (Dintenfass, 1964a). One of the most potent causes of an alteration in viscosity is the hæmatocrit value, especially at high shear rates. High hæmoglobin values will be associated with higher viscosity ranges; possibly related to the reduced viscosity of anæmia is the low incidence of occlusive vascular disease in premenopausal women (after the menopause the incidence is similar to that in men). Whether the cholesterol (and other fat) level of serum has any influence on viscosity is uncertain (Shinagawa, 1964). It is possible that high levels of cholesterol initiate excessive boundary layer separation by increasing blood viscosity, and that its excessive local deposition is only a secondary phenomenon.

The recent finding that a high sugar consumption may increase the atheromatous tendency (Yudkin and Roddy, 1964) may have its basis in an alteration of plasma viscosity following a high sugar intake. The alteration in viscosity of water when sugar is added is well known, but the effect on plasma viscosity of comparatively small alterations in blood sugar has not, to our knowledge, been investigated. This theory offers a simple alternative to the previous one postulating a defect of the whole metabolic state of the organism.

If this theory is correct, then the effect of other factors that alter viscosity may be predicted, regarding their atherogenic potency. An increase in viscosity will give rise to an increase in the stability of the stagnant zone, and will tend to increase its size.

From the effect of Reynolds number on boundary layer separation it would be expected that alterations in flow velocities would have similarly predictable consequences. Factors that are thought to have an important bearing on the development of atheroma are stress and exercise. The effect of exercise on the circulation is to increase flow volumes; in effect, this is an increase in flow velocity. The structures most likely to be affected by exercise are the heart and the legs (considering "exercise" in its usually accepted meaning). Even if there was an increased tendency to stagnant deposition in arteries because of viscosity alterations, regular and fairly frequent increases of flow rates as a result of exercise would introduce violent turbulence into the static areas, resulting in clearing of any recently accumulated loosely-attached debris.

One of the regions where this would be less likely is in the cerebral circulation, where the peripheral resistance is thought to be constant, and flow rates less variable than elsewhere in the body. Cerebral 
vascular disease is much less a disease of the sedentary than is coronary disease, but appears to have a relation to polycythæmia (J. A. Acheson, personal communication, 1965) where serum viscosity would be raised.

The effect of "stress" on circulatory flow rates is much less predictable. Presumably, stress results in increased liberation of adrenaline, which in turn produces constriction of small vessels, with its tendency to increase blood pressure, etc. A similar vasoconstriction, produced as a result of a less clearly defined stimulus, possibly of renal origin, is thought to be the cause of essential hypertension. Such a vasoconstriction, especially if of unequal amount in differing parts of the body, could have a marked effect at the mouths of the larger vessels, due to the alteration of pressure gradient in these places.

As boundary-layer separation is very closely governed by the actual size and shape of vascular branches, it is wondered whether a process similar to atheromatous development is responsible for the adjustment of arterial size to local flow demands. Osborn (1963) cited examples of apparent atheromatous change in the vessels of infants and small children, and it was suggested (Hugh and Fox, 1963) that this might be a physiological process of adjustment rather than a pathological one. One of the ways of providing a system free of boundary layer separation zones is to adjust the calibre of branches to suit the amount of flow within them. Even if this were done for any particular flow state, then an alteration in the amount of flow would alter the conditions. In the body, in health, flow rates vary constantly, because of pulsatility and variations in physiological requirements (shunting of blood in various areas because of exercise, taking food, sleep, etc.).

Attention has recently been drawn (Ross Russell, 1963) to the occurrence of micro-emboli in relation to arterial stenoses, in particular to those found at the origin of the internal carotid artery. The emboli formed may have a varying composition (David, et al., 1963). The phenomena related to these emboli have for some time been thought to be similar to those which can herald the onset of a major cerebral catastrophe ("stuttering" onset of a hemiplegia). Similarly, small areas of infarction are known to occur distal to a renal artery stenosis.

Once a stenosis forms in an artery, the velocity of flow across it will increase and prevent a rapid build up of further deposits. Immediately beyond the stenosis, however, where the flow profile expands, there will be areas of quite marked stagnation, and the formation of deposits will be facilitated, if other conditions are suitable (platelets, fibrin, and cholesterol, etc.). Once deposits reach a critical size they produce their own effect on flow. As boundary layer separation is an unsteady oscillating phenomenon, the instability promotes detachment of any loosely attached particles. If these are aggregates of blood constituents they may be sufficiently integrated to pass on without breaking up to become lodged in the appropriate distal vessel.

The absence of atheroma in the venous system may also be explained by this hypothesis. Boundary layer separation, and stasis zones, occur where arterial branching produces a net increase in the area of flow. In the venous system, the junction of veins results in a corresponding reduction in flow area, so that velocities will tend to increase, thus promoting the stability of the boundary layer and reducing the likelihood of zones of stasis.

One of the major abnormalities arising from this hypothesis as a possible cause of atheroma is alteration in blood viscosity, and it is suggested that more attention be given to measuring this; the development of suitable techniques which give results that indicate intravascular conditions will probably require a large part of the effort. When suitable techniques are available, they should be directed not only to the patients who already exhibit signs of atheromatous development, but to those in whom such development is likely, together with a suitable "control" population. A study of the effect on blood viscosity of varying contents of cholesterol, sugar, etc., might be the most rapid means of confirming this theory, which could lead to a reorientation of thought and investigation into the problem of atheroma.

\section{SUMMARY}

The coincidence between the sites where atheroma commonly forms and the zones where in a flow system local stasis is produced due to boundary layer separation appears to support the view that atheroma is due to a process of deposition, or thrombosis, from the circulating blood.

This paper considers the factors controlling boundary layer separation, and presents pictures of flow experiments carried out to confirm the location of the zones of stasis produced by the separation.

Some of the factors known to be related to atherogenesis are considered from the way in which they might affect the process of boundary layer separation. The viscosity of the circulating fluid is suggested to be the probable main factor controlling the deposition of atheroma; and further investigation of the effect on viscosity of various blood constituents appears desirable. 
We wish to thank Professor R. H. Evans, Department of Civil Engineering and Professor A. S. Johnstone, Department of Radiodiagnosis, both of the University of Leeds, for their encouragement of the collaboration which led to this paper.

Dr. R. Y. Keers kindly read the original manuscript, and we are indebted to him for his helpful criticism and advice.

\section{REFERENCES}

Corcoran, A. C., Lewis, L. A., Dustan, H. P., and Page, I. H. (1956). Atherosclerotic complications in hypertensive disease. Ann N.Y. Acad. Sci., 64, 620.

David, N. J., Klintworth, G. K., Friedberg, S. J., and Dillon, M. (1963). Fatal atheromatous cerebral embolism associated with bright plaques in the retinal arterioles. Neurology (Minneap.), 13, 708.

Dintenfass, L. (1964a). Rheologic approach to thrombosis and atherosclerosis. Angiology, 15, 333.

- (1964b). A trolley viscometer for estimating viscosity and clotting of blood in hospital wards. Lancet, 2 567.

Duguid, J. B. (1955). Mural thrombosis in arteries. Brit. med. Bull., 11, 36.

Duncan, L. E., Cornfield, J., and Buck, K. (1962). The effect of blood pressure in the passage of labeled plasma albumin into canine aortic wall. $\mathcal{f}$. clin. Invest., 41, 1537.

Harkness, J. (1963). A new instrument for the measurement of plasma-viscosity. Lancet, 2, 280.
Hess, R. (1964). Evaluation of drugs active against experimental atherosclerosis. In Advances in Lipid Research, ed. R. Paoletti and D. Kritchewsky, Vol. 2, p. 295. Academic Press, New York.

Hugh, A. E., and Fox, J. A. (1963). Hypotensive anæsthesia. Lancet, 2, 247.

Masson, G. M. C., McCormack, L. J., Dustan, H. P., and Corcoran, A. C. (1958). Hypertensive vascular disease as a consequence of increased arterial pressure. Amer. F. Path., 34, 817.

Mitchell, J. R. A., and Schwartz, C. J. (1965). Arterial Disease. Blackwell Scientific Publications, Oxford.

Osborn, G. R. (1963). The Incubation Period of Coronary Thrombosis. Butterworth, London.

Page, I. H. (1954). Atherosclerosis (Lewis A. Connor Memorial Lecture.) Circulation, 10, 1.

Rokitansky, C. (1844). Handbuch der pathologischen Anatomie. Braumüller, Vienna. See $A$ Manual of Pathological Anatomy transl. Geo. E. Day, (1852), Vol. 4, pp. 261-272. Sydenham Society, London.

Ross Russell, R. W. (1963). Atheromatous retinal embolism. Lancet, $2,1354$.

Schettler, G. (1961). Arteriosklerose. Thieme, Stuttgart.

Shinagawa, T. (1964). Studies on atherosclerosis, mainly about its relationship with serum viscosity. $\mathrm{fap}$. Circulat. F., 28, 324.

Texon, M. (1960). The hemodynamic concept of atherosclerosis. Bull. N.Y. Acad. Med., 36, 263.

Yudkin, J., and Roddy, J. (1964). Levels of dietary sucrose in patients with occlusive atherosclerotic disease. Lancet, 2, 6. 